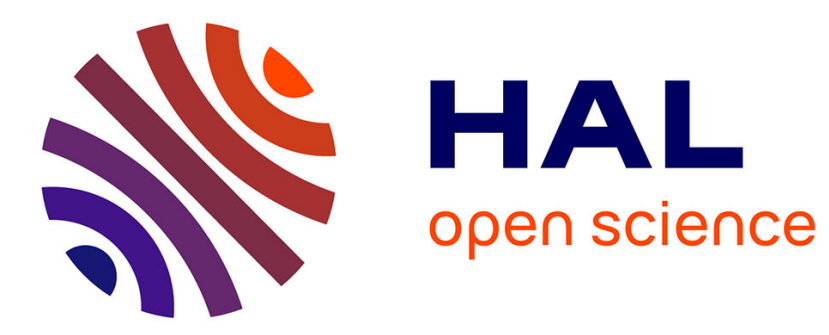

\title{
Scaling laws of impact induced shock pressure and particle velocity in planetary mantle
}

\author{
J. Monteux, J. Arkani-Hamed
}

\section{To cite this version:}

J. Monteux, J. Arkani-Hamed. Scaling laws of impact induced shock pressure and particle velocity in planetary mantle. Icarus, 2016, 264, pp.246-256. 10.1016/j.icarus.2015.09.040 . hal-01637422

\author{
HAL Id: hal-01637422 \\ https://hal.uca.fr/hal-01637422
}

Submitted on 18 Jan 2018

HAL is a multi-disciplinary open access archive for the deposit and dissemination of scientific research documents, whether they are published or not. The documents may come from teaching and research institutions in France or abroad, or from public or private research centers.
L'archive ouverte pluridisciplinaire HAL, est destinée au dépôt et à la diffusion de documents scientifiques de niveau recherche, publiés ou non, émanant des établissements d'enseignement et de recherche français ou étrangers, des laboratoires publics ou privés. 


6

7

8

\section{Scaling Laws of Impact Induced Shock Pressure and Particle Velocity in Planetary Mantle}

\section{J. Monteux}

Laboratoire Magmas et Volcans, Clermont-Ferrand, France

\section{J. Arkani-Hamed}

Department of Physics, University of Toronto, Toronto, Canada

Department of Earth and Planetary Sciences, McGill University, Montreal, Canada

Abstract. While major impacting bodies during accretion of a Mars type planet have very low velocities $(<10 \mathrm{~km} / \mathrm{s})$, the characteristics of the shockwave propagation and, hence, the derived scaling laws are poorly known for these low velocity impacts. Here, we use iSALE-2D hydrocode simulations to calculate shock pressure and particle velocity in a Mars type body for impact velocities ranging from 4 to $10 \mathrm{~km} / \mathrm{s}$. Large impactors of 100 to $400 \mathrm{~km}$ in diameter, comparable to those impacted on Mars and created giant impact basins, are examined. To better represent the power law distribution of shock pressure and particle velocity as functions of distance from the impact site at the surface, we propose three distinct regions in the mantle: a near field regime, which extends to 1-3 times the projectile radius into the target, where the peak shock pressure and particle velocity decay very slowly with increasing distance, a mid field region, which extends to $\sim 4.5$ times the impactor radius, where the pressure and particle velocity decay exponentially but moderately, and a more distant far field region where the pressure and particle velocity decay strongly with distance. These scaling laws are useful to determine impact heating of a growing proto-planet by numerous accreting bodies.

9

0

1


Introduction:

Small planets are formed by accreting a huge number of planetesimals, a few km to a few tens of $\mathrm{km}$ in size, in the solar nebula [e.g., Wetherill and Stewart, 1989; Matsui, 1993: Chambers and Wetherill, 1998; Kokubo and Ida, 1995, 1996, 1998, 2000; Wetherill and Inaba, 2000; Rafikov, 2003; Chambers, 2004; Raymond, et al., 2006]. An accreting body may generate shock wave if the impact-induced pressure in the target exceeds the elastic Hugoniot pressure, $\sim 3 \mathrm{GPa}$, implying that collision of a planetesimal with a growing planetary embryo can generate shock waves when the embryo's radius exceeds $150 \mathrm{~km}$, assuming that impact occurs at the escape velocity of the embryo and taking the mean density of the embryo and projectile to be 3000 $\mathrm{kg} / \mathrm{m}^{3}$. Hundreds of thousands of collisions must have occurred during the formation of small planets such as Mercury and Mars when they were orbiting the Sun inside a dense population of planetesimal. Such was also the case during the formation of embryos that later were accreted to produce Venus and Earth. Terrestrial planets have also experienced large high velocity impacts after their formation. Over 20 giant impact basins on Mars with diameters larger than $1000 \mathrm{~km}$ [Frey, 2008)], the Caloris basin on Mercury with a $1550 \mathrm{~km}$ diameter, and the South Pole Aitken basin on Moon with a $2400 \mathrm{~km}$ diameter are likely created during catastrophic bombardment period at around $4 \mathrm{Ga}$. The overlapping Rheasilvia and Veneneia basins on 4-Vesta are probably created by projectiles with an impact velocity of about $5 \mathrm{~km} / \mathrm{s}$ within the last 1-2 Gy [Keil et al., 1997; Schenk et al., 2012].

The shock wave produced by an impact when the embryo is undifferentiated and completely solid propagates as a spherical wave centered at the impact site until it reaches the surface of the embryo in the opposite side. Each impact increases the temperature of the embryo within a region near the impact site. Because impacts during accretion occur from different directions, the mean temperature in the upper parts of the embryo increases almost globally. On the other hand, the shock wave produced by a large impact during the heavy bombardment period must have increased the temperature in the mantle and the core of the planets directly beneath the impact site, enhancing mantle convection [e.g., Watters et al., 2009; Roberts and Arkani-Hamed, 2012, 
2014], modifying the CMB heat flux which could in turn favour a hemispheric dynamo on Mars [Monteux et al., 2015], or crippling the core dynamo [e.g., Arkani-Hamed and Olson, 2010a].

The impact-induced shock pressure inside a planet has been investigated by numerically solving the shock dynamic equations using hydrocode simulations [e.g., Pierazzo et al., 1997; Wuennemann and Ivanov, 2003; Wuennemann et al., 2006; Bar and Citron, 2011; Kraus et al., 2011; Ivanov et al., 2010; Bierhaus et al., 2012] or finite difference techniques [e.g., Ahrens and O’Keefe, 1987; Mitani, 2003]. However, these numerical solutions demand considerable computer capacity and time and are not practical for investigating the huge number of impacts that occur during the growth of a planet. Hence, the scaling laws derived from field experiments [e.g., Perret and Bass, 1975; Melosh, 1989] or especially from hydrocode simulations [Pierazzo et al., 1997] are of great interest when considering the full accretionary history of a planetary objects [e.g. Senshu et al., 2002, Monteux et al., 2014] or when measuring the influence of a single large impact on the long-term thermal evolution of deep planetary interiors [e.g. Monteux et al., 2007, 2009, 2013, Ricard et al, 2009; Roberts et al., 2009; Arkani-Hamed and Olson, 2010a; Arkani-Hamed and Ghods, 2011]. Although the scaling laws provide approximate estimates of the shock pressure distribution, their simplicity and the small differences between their results and those obtained by the hydrocode simulations of the shock dynamic equations (that are likely within the numerical errors that could have been introduced due to the uncertainty of the physical parameters used in the hydrocode models) make them a powerful tool that can be combined with other geophysical approaches such as dynamo models [e.g. Monteux, et al., 2015] or convection models [e.g. Watters et al, 2009; Roberts and Arkani-Hamed, 2012, 2014].

During the decompression of shocked material much of the internal energy of the shock state is converted into heat leading to a temperature increase below the impact site. The present study focuses on deriving scaling laws of shock pressure and particle velocity distributions in silicate mantle of a planet on the basis of several hydrocode simulations. The scaling laws of Pierazzo et al. [1997] were derived using impact velocities of 10 to $80 \mathrm{~km} / \mathrm{s}$, hence may not be viable at low impact velocities. For example, at an impact velocity of $5 \mathrm{~km} / \mathrm{s}$, comparable to the escape velocity of Mars, the shock pressure scaling law provides an unrealistic shock pressure that increases with depth. Here we model shock pressure and particle velocity distributions in the mantle using hydrocode simulations for impact velocities of 4 to $10 \mathrm{~km} / \mathrm{s}$ and projectile 
diameters ranging from 100 to $400 \mathrm{~km}$, as an attempt to extend Pierazzo et al.'s [1997] scaling laws to low impact velocities and reasonable impactor radii occurring during the formation of terrestrial planets. Hence, on the basis of our scaling laws it is possible to estimate the temperature increase as a function of depth below the impact site for impact velocities compatible with the accretionary conditions of terrestrial protoplanets. These scaling laws can easily be implemented in a multi-impact approach [e.g. Senshu et al, 2003, Monteux et al., 2014] to monitor the temperature evolution inside a growing protoplanet whereas it is not yet possible to adopt hydrocode simulations for that purpose.

The hydrocode models we have calculated are described in the first section, while the second section presents the scaling laws derived from the hydrocode models. The concluding remarks are relegated to the third section.

\section{Hydrocode models of shock pressure distribution:}

The huge number of impacts during accretion makes it impractical to consider oblique impacts. Not only it requires formidable computer time, but more importantly because of the lack of information about the impact direction, i.e. the impact angle relative to vertical and azimuth relative to north. Therefore, we consider only head-on collisions (vertical impact) to model the thermo-mechanical evolution during an impact between a differentiated Mars size body and a large impactor. We use the iSALE-2D axisymmetric hydrocode, which is a multi-rheology, multi-material hydrocode, specifically developed to model impact crater formation on a planetary scale [Collins et al., 2004, Davison et al., 2010]. To limit computation time, we use a 2 $\mathrm{km}$ spatial resolution (i.e. more than 25 cells per projectile radius, cppr) and a maximum time step of $0.05 \mathrm{~s}$ which is sufficient to describe the shockwave propagation through the entire mantle. The minimum post impact monitoring time is set to the time needed by the shockwave to reach the core-mantle boundary $(\approx 5$ minutes for the impact velocities studied here).

We investigate the shock pressure and particle velocity distributions inside a Mars size model planet for impact velocities $V_{\mathrm{imp}}$ of 4 to $10 \mathrm{~km} / \mathrm{s}$ and impactors of 100 to $400 \mathrm{~km}$ in diameter. Such impactors are capable of creating basins of 1000 to $2500 \mathrm{~km}$ in diameter according to 
Schmidt and Housen [1987] and Holsapple [1993] scaling relationships between the impactor diameter and the resulting basin diameter. These basins are comparable with the giant impact basins of Mars created during the heavy bombardment period at around 4 Ga [Frey, 2008].

In our models, the impactor was simplified to a spherical body of radius $R_{\mathrm{imp}}$ with uniform composition while the target was simplified to a two layers spherical body of radius $R$ and an iron core radius of $R_{\text {core. }}$. The silicate mantle has a thickness of $\delta_{\mathrm{m}}$ (See Table 1). We adopt physical properties of silicates (dunite or peridotite) for both the mantle and the impactor to monitor the shock pressure and the particle velocity in a Mars type body. We approximate the thermodynamic response of both the iron and silicate material using the ANEOS equation of state [Thompson and Lauson, 1972, Benz et al., 1989]. To make our models as simple as possible we do not consider here the effects of porosity, thermal softening or low density weakening. However, as a first step towards more realistic models, we investigate the influence of acoustic fluidization and damage. All these effects can be accounted for in iSALE-2D and we will consider each effect in a separate study in near future.

The early temperature profile of a Mars size body is difficult to constrain because it depends on its accretionary history, on the amount of radiogenic heating and on the mechanisms that led to its core formation [e.g., Senshu et al., 2002]. The uncertainties on the relative importance of these processes as well as the diversity of the processes involved in the core formation lead to a wide range of plausible early thermal states after the full differentiation of Mars. Since we do not consider here the thermal softening during the impact, we assumed the same radially dependent preimpact Martian temperature field in all our simulations. Fig. 1 shows the pre-impact temperature profile as a function of depth. As the pre-impact pressure is governed by the material repartition and as we consider a differentiated Mars, the pre-impact pressure is more straightforward. Fig. 1 also shows the pre-impact hydrostatic pressure used in our models as a function of depth considering a $1700 \mathrm{~km}$ thick silicate mantle surrounding a $1700 \mathrm{~km}$ radius iron core. We emphasize that the peak pressure shown in our study does not include this hydrostatic pressure. However, the hydrostatic pressure is taken into consideration in calculating the hydrocode models. The peak pressure presents the shock induced pressure increase and is expected to depend on the physical properties of the target, but not on the size of the target as long as the size is large enough to allow shock wave propagates freely without interference with 
reflected waves. A scaling law should reflect the properties of the shock wave propagation in a uniform media. Fig. 2 shows the typical time evolution of the compositional and pressure fields after a $100 \mathrm{~km}$ diameter impact with $V_{\mathrm{imp}}=10 \mathrm{~km} / \mathrm{s}$. Immediately after the impact, the shockwave propagates downward from the impact site. The shock front reaches the core-mantle boundary in less than 5 minutes while the transient crater is still opening at the surface. It is worth mentioning that the main goal is to derive a scaling law which is useful for numerous impacts during accretion where no information is available about the impact direction, i.e. the impact angle relative to vertical and azimuth relative to north. Moreover, the pressure reduction near the surface due to interference of the direct and reflected waves can easily be accommodated following the procedure by Melosh [1989], which is adapted to spherical surface by ArkaniHamed [2005], when applying the scaling law to a particular accretion scenario.

In Fig. 3, we monitor the peak pressure as a function of the distance from the impact site $d$ normalized by the impactor radius $R_{\text {imp }}$ along the symmetry axis for the case illustrated in Fig. 2. In our iSALE models, the impact-induced pressure fields (as well as temperature and velocity fields) are extracted from a cell-centered Eulerian grid data. To validate our models, we have tested different spatial resolutions expressed here in terms of cells per projectile radius (cppr). In Fig. 3a, we represent the peak pressure decrease as a function of depth for cppr values ranging from 5 to 50, showing convergence for cppr values larger than 25. As illustrated in Fig. 3a, the difference between the results with 25 and $50 \mathrm{cppr}$ is less than 10\%. This resolution study is in agreement with Pierazzo et al., [2008] who have shown that the iSALE models converge for resolutions of $20 \mathrm{cppr}$ or higher, although a resolution of $10 \mathrm{cppr}$ still provides reasonable results (a resolution of $20 \mathrm{cppr}$ appears to underestimate peak shock pressures by at most 10\%). The resolution is 25 cppr or higher in our models.

In Pierazzo et al., [1997], the impactor radius ranged between 0.2 and $10 \mathrm{~km}$. In Fig. 3b, we compare our results obtained with $R_{\mathrm{imp}}=10 \mathrm{~km}, R_{\mathrm{imp}}=50 \mathrm{~km}$ and the results obtained by Pierazzo et al., [1997]. Fig. 3b shows that even with a radius of $50 \mathrm{~km}$, both our results and the results from Pierazzo et al., [1997] are in good agreement, confirming that the impactor size has minor effects on the peak pressure evolution with depth. The small differences between our results with different impactor radii (discussed further in more details) are plausibly the direct consequence of using increasing cppr values with increasing impactor radii. Consequently, we will use the 


$$
P=P_{o}\left(d / R_{\mathrm{imp}}\right)^{n}, \text { for } d>1-3 \text { times } R_{\mathrm{imp},} \text { and } n=-1.25-0.625 \log \left(V_{\mathrm{imp}}\right) \text {, }
$$
and our results diverge from the results obtained by Pierazzo et al., [1997].

\section{Shock pressure and particle velocity scaling laws at low impact velocities:} pressure decays exponentially as

normalized distance in all of our figures, as equations of motion should be invariant under rescaling of distance [Melosh, 1989, Pierazzo et al, 1997]. However, to monitor the peak pressure evolution with $R_{\mathrm{imp}}=10 \mathrm{~km}$ and to maintain a reasonable computational time, we have used only $10 \mathrm{cppr}$. This figure confirms that below $10 \mathrm{cppr}$, the spatial accuracy is insufficient

A given hydrocode simulation may take on the order of 48 hours to determine a $2 \mathrm{D}$ shock pressure and particle velocity distributions in the mantle of our model planet. The impact velocity is about $4 \mathrm{~km} / \mathrm{s}$ for a protoplanet with a radius of $2860 \mathrm{~km}$ and mean density of 3500 $\mathrm{kg} / \mathrm{m}^{3}$, assuming that impacts occur at the escape velocity of the protoplanet. Mars is more likely a runaway planetary embryo formed by accreting small planetesimals and medium size neighboring planetary embryos. This indicates that the accreting bodies had velocities higher than $4 \mathrm{~km} / \mathrm{s}$ when Mars was growing from $2860 \mathrm{~km}$ radius to its present radius of about 3400 $\mathrm{km}$. Taking the mean radius of the impacting bodies to be $100 \mathrm{~km}$, which is larger than that of a typical planetesimal, more than 15,000 bodies must have accreted at impact velocities higher than $4 \mathrm{~km} / \mathrm{s}$. Calculating the impact induced shock pressure and particle velocity inside the growing Mars would be formidable if hydrocode simulation is adopted for each impact. Because the impact-induced shock pressure $P$ and particle velocity $V_{\mathrm{p}}$ inside a planetary mantle decease monotonically with distance from the impact side, simple exponential functions have been proposed to estimate peak pressure and particle velocity in the mantle of an impacted body. Solving the shock dynamic equations by a finite difference technique for silicate target and projectile, Ahrens and O'Keefe [1987] showed that pressure distribution in the target displays three regimes: an impedance match regime, Regime I, which extends to 1-3 times the projectile radius into the target where the peak shock pressure is determined by the planar impedance match pressure [McQueen et al., 1970]; a shock pressure decay regime, Regime II, where the 
and the elastic regime, Regime III, where the shock pressure is reduced below the strength of target, the Hugoniot elastic limit, and the shock wave is reduced to an elastic wave with pressure decaying as $d^{-3}$. In equation (1) $d$ is the distance from the impact site at the surface, $R_{\text {imp }}$ is the projectile radius, and $V_{\text {imp }}$ is the impact velocity in $\mathrm{km} / \mathrm{s}$. The peak pressure measurements in the nuclear explosions [Perret and Bass, 1975] led Melosh [1989] to propose a scaling low for the particle velocity distribution assuming conservation of momentum of the material behind the shock front which is taken to be a shell of constant thickness. Using several different target materials, and adopting hydrocode simulations Pierazzo et al. [1997] showed that the shock pressure $P$ and particle velocity $V_{\mathrm{p}}$ decrease slowly in the near field zone, but rapidly in the deeper region,

$$
\begin{array}{lll}
P=P_{\text {ic }}\left(d_{\text {ic }} / d\right)^{n}, & n=-1.84+2.61 \log \left(V_{\text {imp }}\right), & d>d_{\text {ic }} \\
V_{\mathrm{p}}=V_{\text {pic }}\left(d_{\text {ic }} d d\right)^{m} & m=-0.31+1.15 \log \left(V_{\text {imp }}\right), & d>d_{\text {ic }}
\end{array}
$$

The authors coined an isobaric zone of shock pressure $P_{\text {ic }}$ and particle velocity $V_{\text {pic }}$ for the near field of radius $d_{\mathrm{ic}}$, about $1.5 R_{\mathrm{imp}}$. Equations $2 \mathrm{a}$ and $2 \mathrm{~b}$ were derived by averaging results from many different materials and impactor sizes. The impact velocities adopted were 10 to $80 \mathrm{~km} / \mathrm{s}$ and the projectile diameter ranged from 0.4 to $20 \mathrm{~km}$.

In a log-log plot the peak shock pressure and the corresponding particle velocity are linear functions of distance from the impact site,

$$
\begin{aligned}
& \log P=a+n \log \left(d / R_{\mathrm{imp}}\right) \\
& \log V_{\mathrm{p}}=c+m \log \left(d / R_{\mathrm{imp}}\right)
\end{aligned}
$$

where $a$ is the logarithm of pressure and $c$ is the logarithm of particle velocity both at $R_{\text {imp }}$ and $n$ and $m$ are decay factors. All parameters are impact velocity dependent:

$$
\begin{aligned}
& a=\alpha+\beta \log \left(V_{\mathrm{imp}}\right) \\
& \mathrm{c}=\gamma+\Omega \log \left(V_{\mathrm{imp}}\right)
\end{aligned}
$$


247

248

249

250

251

252

253

254

255

256

257

258

259

260

261

262

263

264

265

266

267

268

269

270

271

272

273

274

275

276

277

and

$n=\lambda+\delta \log \left(V_{\mathrm{imp}}\right)$

$m=\eta+\zeta \log \left(V_{\mathrm{imp}}\right)$

Figure 4 shows the peak shock pressure inside the mantle of the model planet we obtained by hydrocode simulation and using a projectile of $100 \mathrm{~km}$ diameter at $10 \mathrm{~km} / \mathrm{s}$ impact velocity. There is actually no isobaric region, rather the peak pressure decays slowly in the near field zone but much rapidly in the deeper parts, similar to the results by Ahrens and O'Keefe [1987]. Although the regression lines fitted to the near field and far field are good representatives of the shock pressure distribution, they intersect at a much higher pressure than that of the hydrocode model and overestimate the pressure by as much as $30 \%$ in a large region located between the near field and the far field. Therefore, to better approximate the pressure distribution we fit the pressure curve by three lines, representing the near field, mid field and far field regions, which render a much better fit as seen in Figure 4.

Figure 5a shows the peak shock pressure versus distance from the impact site for impact velocities of 4 to $10 \mathrm{~km} / \mathrm{s}$ and an impactor of $100 \mathrm{~km}$ in diameter. Because of different phenomenon such as excavation, melting, vaporization and intermixing between the target and projectile material, the shock front in near field is more difficult to characterize by our numerical models even for high cppr. Also, as the shock wave is not yet detached from the impactor, it cannot be treated as a single shock wave. Hence, the near field - mid field boundary and the scaling laws for the near field are less accurate than for the two other fields especially for large impact velocities $\left(V_{\mathrm{imp}}>7 \mathrm{~km} / \mathrm{s}\right)$. In Figure 5, the larger dots show the intersections of the linear regression lines. For example a dot that separates near field from mid field is the intersection of the regression lines fitted to the near field and midfield. The regression lines are determined from fitting to the hydrocode data. Visually, we first divide the hydrocode data of a given model into three separate sections with almost linear trends, and then fit the regression lines to those three trends. Figure 5a shows the hydrocode data, small dots, and the regression lines, demonstrating the tight fitting of the lines to the data. The large dots in the figure show the intersection of the regression lines of the adjacent regions. For example the dot that shows the near-field mid-field boundary is the intersection of the regression lines fitted to the near field and 
mid field hydrocode data. Note that a big dot does not necessarily coincide with the exact hydrocode result, a small dot. As the near field - mid field boundary is relatively less resolved, Figure 5a shows a scatter of the dots separating those two fields and a slight slope change from $V_{\text {imp }}>7 \mathrm{~km} / \mathrm{s}$. In the average the near field - mid field boundary is at $\sim 2.3 R_{\text {imp }}(\sim 115 \mathrm{~km})$ from the impact site. Figure 5a shows that the depth to the mid field - far field boundary is almost independent of the pressure. It is at about $4.5 R_{\text {imp }}(\sim 225 \mathrm{~km})$ from the impact site.

We propose three scaling laws for shock pressure, and three for particle velocity:

$\log P_{i}=a_{i}+n_{i} \log \left(d_{i} / R_{\text {imp }}\right), \quad i=1,2$, and 3.

$\log V_{p i}=c_{i}+m_{i} \log \left(d_{i} / R_{\text {imp }}\right), \quad i=1,2$, and 3 .

For the near field $d_{i}<2.3 R_{\text {imp }}$, mid field $2.3 R_{\text {imp }}<d_{i}<4.5 R_{\text {imp }}$, and far field $d_{i}>4.5 R_{\text {imp. Table }}$ 2 lists the values of the parameters in the above equations as well as the misfits obtained from our regressions (smaller than 0.001 in all the regressions calculated here). Figure 5a shows the three regression lines fitted to the near field, mid field, and far field of each model for shock pressure, and Figure 5b displays those for particle velocity. The misfits from Table 2 are based on the fixed end points, shown as dots in Fig. 5.

The close agreement between Pierazzo et al. [1997] model for impact velocity of $10 \mathrm{~km} / \mathrm{s}$ derived by averaging results of impactors with diameters 0.4 to $20 \mathrm{~km}$, and our result for the same impact velocity but an impactor of $100 \mathrm{~km}$ in diameter indicates that the shock pressure distribution is less sensitive to impactor size in a log-log plot of pressure versus distance normalized to the impactor radius. To further investigate this point, we calculate models with impactor sizes of 100 to $400 \mathrm{~km}$ in diameter. Figure 6a shows the hydrocode results for impact velocity of $10 \mathrm{~km} / \mathrm{s}$ using different impactor size. The result obtained for $R_{\text {imp }}=10 \mathrm{~km}$ is not included in the figure because of its low cppr value (see Fig. 3b). The curves have almost the same slope in the far field, and small deviations in the mid field and near field.

Pierazzo et al. [1997] used several different rock types for the solid mantle and concluded that their scaling laws are less sensitive to the rock types. Bearing in mind that dunite and peridotite are probably the most representative rocks for solid mantle, we run a hydrocode model using an 
impactor of $100 \mathrm{~km}$ in diameter, impact velocity of $10 \mathrm{~km} / \mathrm{s}$, and peridotite as a representative mantle and impactor rock. Figure $6 \mathrm{~b}$ compares the results for the dunite and peridotite models. They are indeed very similar, especially in the far field region, where the exponential factor $n$ in Equation (3a) is -1.449 for dunite and -1.440 for peridotite. The major differences between the two models arise from the near field zone, hence propagates down to the deeper regions.

Among the other parameters and phenomenon that may influence the shockwave propagation (porosity, thermal softening...), accounting for the acoustic fluidization is required to accurately simulate the formation of a complex crater [e.g., Melosh, 1979, Bray et al., 2008, Potter et al, 2012]. Indeed, acoustic fluidization is invoked to explain the collapse of complex craters by modifying the frictional strength of the damaged target. Figure $6 \mathrm{c}$ compares the results obtained for $R_{\mathrm{imp}}=50 \mathrm{~km}$ and $V_{\mathrm{imp}}=10 \mathrm{~km} / \mathrm{s}$ considering acoustic fluidization and an Ivanov damage model which prescribes damage as a function of plastic strain. This figure shows that for the near and mid fields, the results are similar. Pierazzo et al. [1997] did not include acoustic fluidization or damage in their models. Hence, our results without acoustic fluidization or damage model and the results from Pierazzo are in good agreement (Fig. 6c). As soon as the far field is reached, acoustic fluidization and damage tend to reduce the intensity of the shock pressure. This indicates that building more sophisticated models will be necessary in the near future. As the impact heating is mainly localized in the near- and mid-fields, including a damage model or acoustic fluidization should only weakly affect the thermal evolution of a growing protoplanet. However, it is worth mentioning that we are not concerned with the shape of the crater produced by a large impact, rather the main goal of our study is to extend the scaling laws of Pierazzo et al., [1997] to lower impact velocities which are more compatible with accretionary conditions.

Figure 7 shows the impact velocity dependence of $a, n, c$, and $m$. Also included in Figure $7 \mathrm{~b}$ is the model by Ahrens and O'Keefe [1987] which was derived using impact velocities of $5 \mathrm{~km} / \mathrm{s}$ and higher. Pierazzo et al. [1997] used impact velocities higher than those considered in the present study, except for their $10 \mathrm{~km} / \mathrm{s}$ model. Hence, their results are shown in Figure $7 \mathrm{~b}$ by only one point, asterisk, at the impact velocity of $10 \mathrm{~km} / \mathrm{s}$.

The shock pressure along a non-vertical profile is not supposed to be the same as the one along a 
vertical profile, largely because of the pre-impact lithostatic pressure. As emphasized by Pierazzo et al. [1997, 2008] the shock front in deeper regions appears relatively symmetric around the impact point. Of course, it is not realistic in the case of an oblique impact (not studied here) and for the shallowest angles where the surface significantly affects the shock pressure decay. We have monitored the effect of the shockwave propagation angle $\theta$ with values varying between $90^{\circ}$ (vertical profile) to $27^{\circ}$ (Fig. 8). Similarly to Pierazzo et al [1997], we did not find a significant angle dependence on our results especially when $\theta$ is ranging between $90^{\circ}$ and $45^{\circ}$. For smaller values of $\theta$, the surface effects appear to modify the shockwave propagation by reducing its intensity (Fig. 8 a). Except in the mid field, where the $n$ value decreases from -0.6 to -1.31 , and in the far field, where the $a$ value decreases from 2.54 to 1.93 , the coefficients $a$ and $n$ from our scaling laws do not change significantly with the angle (Fig. 8 b). This is particularly true in the near field where most of the impact heating occurs.

Scaling laws have been used by many investigators [e.g., Senshu et al., 2002; Tonks and Melosh, 1992, 1993; Watters et al, 2009; Roberts et al., 2009; Arkani-Hamed and Olson, 2010a, 2010b; Arkani-Hamed and Ghods, 2011], mainly because they require a much smaller computer and much less computer time and the difference between a hydrocode model and a corresponding scaling model is minute. For example, Figure 9 shows the 2D distribution of the peak shock pressure determined for an impactor of $100 \mathrm{~km}$ in diameter and an impact velocity of $10 \mathrm{~km} / \mathrm{s}$ calculated using our scaling laws in near field, mid field, and far field, and the parameter values from Table 2. The grid spacing is $2 \mathrm{~km}$ in radial direction and 0.03 degrees in the colatitude direction. The entire computer time in a PC, CPU: $2.4 \mathrm{GHz}$, was only 16 seconds, which also calculated the 2D distribution of shock-induced temperature increase using Watters et al.'s [2009] foundering shock heating model. The computer time is substantially shorter than 48 hours taken by our corresponding hydrocode model using a CPU: $2.9 \mathrm{GHz}$ laptop. This shows that it is feasible to determine impact heating during the accretion of a terrestrial planet using scaling laws, whereas it is almost impossible to adopt hydrocode simulations for that purpose.

During the decompression of shocked material much of the internal energy of the shock state is converted into heat [O'Keefe and Ahrens, 1977]. Using thermodynamic relations, the waste heat used to heat up the impacted material can be estimated [Gault and Heitowit, 1963; Watters et al., 
2009] and the corresponding temperature increase $\Delta T$ can be obtained. Hence, on the basis of our scaling laws it is possible to estimate the temperature increase as a function of depth below the impact site for impact velocities compatible with the accretionary conditions of terrestrial protoplanets. These scaling laws can easily be implemented in a multi-impact approach [e.g. Senshu et al, 2003, Monteux et al., 2014] to monitor the temperature evolution inside a growing protoplanet whereas it is not yet possible to adopt hydrocode simulations for that purpose. For example, included in Figure 9 is the impact induced temperature increase corresponding to the shock pressure shown in the figure. The temperature increase is determined on the basis of foundering model of Watters et al. [2009] using constant values for the acoustic velocity $C$ (6600 $\mathrm{m} / \mathrm{s}$ ) and the parameter $S(0.86)$ in their expressions:

$\Delta T(P)=\frac{P}{2 \rho_{0} S}\left(1-f^{-1}\right)-(C / S)^{2}[f-\ln f-1]$

$f(P)=-\frac{P}{\beta}\left(1-\sqrt{\frac{2 P}{\beta}+1}\right)^{-1}$

$\beta=\frac{C^{2} \rho_{0}}{2 S}$

with $P$ the shock-increased pressure and $\rho_{0}$ the density prior to shock compression (see Tab. 1 for values).

Due to small size the impactor is not capable of increasing the lower mantle temperature of the model planet significantly, and only minor impact heating of the core has occurred. The thermal evolution model has to be combined to a topographical evolution model to account for the growth of the protoplanet as in Monteux et al., [2014]. In these models, the impact angle (considered here as vertical) probably plays a key role because it influences both the morphology of the impact heating and the shape of the post-impact topography. A more elaborated scaling laws built upon 3D hydrocode models will be developed for that purpose in the near future.

It is worth emphasizing that our scaling laws, like those of others [Ahrens and O'Keefe, 1987; Pierazzo et al., 1997; Mitani, 2003], are derived from a few hydrocode models. Figure 10 shows the profiles of the pressure along the axis of symmetry for comparison. The differences between 
$414 P=\rho V_{p} V_{s}$

415

$$
V_{s}=C+S V_{p}
$$

$$
P=\rho V_{p} V_{s}
$$

\section{Conclusions:}

the hydrocode model and the scaling law are small for the most part, but the exact scaling law differs by $\sim 10 \mathrm{GPa}$ for $d / R_{\mathrm{imp}}=2-3$. This difference arises from the difficulty of correctly describe the near field as previously mentioned. Note that the interpolated model is in much better agreement with the hydrocode model.

A linear relationship has been proposed between the shock wave velocity $V_{s}$ and particle velocity $V_{p}$ on the basis of laboratory measurements [McQueen, 1967; Trunin, 2001]

where $C$ is the acoustic velocity and $S$ is a constant parameter. We estimate the acoustic velocity in the mantle of the model planet on the basis of our hydrocode models (Figure 5a, 5b) using Equation (10) and the Hugoniot equation

where $\rho\left(=3320 \mathrm{~kg} / \mathrm{m}^{3}\right)$ is the pre-shock density. Figure 11 shows the variations of $C$ with depth for models with impact velocities of 4 to $10 \mathrm{~km} / \mathrm{s}$ and an impactor of $100 \mathrm{~km}$ diameter, using $S=1.2$ which is within the values proposed by the authors for dunite [e.g., Trunin, 2001]. Adopting $S=0.86$ [McQueen, 1967] does not change the results significantly, especially in the far field, where the acoustic velocity is less sensitive to particle velocity and linearly increases with depth. However $C$ shows particle velocity dependence in the mid field and near field.

We have modeled the shock pressure and particle velocity distributions in the mantle of a Mars size planet using hydrocode simulations (iSALE-2D) for impact velocities of 4 to $10 \mathrm{~km} / \mathrm{s}$ and projectile diameters ranging from 100 to $400 \mathrm{~km}$. We have extended Pierazzo et al.'s [1997] scaling laws to low impact velocities and also considered large impactor radii occurring during the formation of terrestrial planets. We propose three distinct regions in the mantle: a near field region, which extends to 1-3 times the projectile radius into the target, where the peak shock 
431 pressure and particle velocity decay very slowly with increasing distance, a mid field region, 432 which extends to $\sim 4.5$ times the impactor radius, where the pressure and particle velocity decay 433 exponentially but moderately, and a more distant far field region where the pressure and particle 434 velocity decay strongly with distance. The mid field - far field boundary is well constrained, 435 whereas that of the near field - mid field is a relatively broad transition zone for the impact 436 velocities examined.

Acknowledgements: This research was supported by Agence Nationale de la Recherche 439 (Oxydeep decision No. ANR-13-BS06-0008) to JM, and by Natural Sciences and Engineering Research Council (NSERC) of Canada to JAH. We gratefully acknowledge the developers of iSALE (www.isale-code.de), particularly the help we have received from Gareth S. Collins. We are also grateful to the two reviewers for very helpful suggestions.

443

444

\section{References:}

445

Ahrens, T.J., J.D., O'Keefe, (1987). Impact on the Earth, ocean and atmosphere. Int. J. Impact Eng. 5, 13-32. 122.

447

448

449 450

Arkani-Hamed, J., Magnetic crust of Mars, J. Geophys. Res., 110, E08005, doi:10.1029/2004JE002397, 2005.

Arkani-Hamed, J, and A. Ghods, (2011). Could giant impacts cripple core dynamos of small terrestrial planets?, Icarus 212, 920-934.

Arkani-Hamed, J., Olson, P., (2010a). Giant Impact Stratification of the Martian Core Geophys. Res. Let., 37, L02201, doi:10.1029/2009GL04141

Arkani-Hamed, J., Olson, P., (2010b). Giant impacts, core stratification, and failure of the Martian dynamo. J. Geophys. Res. 115, E07012.

Barr, A., Citron, R., (2011). Scaling of melt production in hypervelocity impacts from highresolution numerical simulations, Icarus 211, 913-916.

Benz, W., Cameron, A.G.W., Melosh, H.J., (1989). The origin of the Moon and the singleimpact hypothesis III. Icarus 81, 113-131. 
Bierhaus, M., K. Wünnemann, and D. Elbeshausen, (2012). Numerical modeling of basinforming impacts: implications for the ehat budget of planetary interiors, 43rd Lunar and Planetary Science Conference. Abstract \# 2174.pdf.

Bray, V. J., Collins, G. S., Morgan, J. V., Schenk, P. M., (2008). The effect of target properties on crater morphology: comparison of central peak craters on the Moon and Ganymede. Meteoritics \& Planetary Science. 43, 1979-1992.

Chambers, J.E., (2004). Planetary accretion in the inner Solar System, Earth Planet. Sci. Lett. $223,241-252$.

Chambers, J.E., and G. W. Wetherill, (1998). Making the terrestrial planets: N-Body integrations of planetary embryos in three dimensions, Icarus, 136, 304-327.

Collins, G. S., H. J. Melosh, and B. A. Ivanov (2004), Modeling damage and deformation in impact simulations, Meteoritics Planet. Sci., 39(2), 217-231, doi:10.1111/j.19455100.2004.tb00337.x.

Davison, T. M., G. S. Collins, and F. J. Ciesla (2010), Numerical modeling of heating in porous planetesimal collisions, Icarus, 208, 468-481, doi:10.1016/j.icarus.2010.01.034.

Frey, H. (2008), Ages of very large impact basins on Mars: Implications for the late heavy bombardment in the inner solar system, Geophys. Res. Lett., 35, L13203, doi:10.1029/2008GL033515.

Gault, D. E., and E. Heitowit (1963), The partition of energy for hypervelocity impact craters formed in rock, paper presented at 6th Hypervelocity Impact Symposium, Firestone Tire and Rubber Co., Cleveland, 30 Apr. to 2 May.

Holsapple, K. A. (1993), The scaling of impact processes in planetary sciences, Annu. Rev. Earth Planet. Sci., 21, 333-373.

Ivanov, B.A., H.J. Melosh, and E. Pierazzo, (2010). Basin-forming impacts: Reconnaissance modeling, Geological Society of America, Special Paper 465, 29-49.

Keil, K., D. Stoffler, S. G. Love, E. R. D. Scott (1997), Constraints on the role of impact heating and melting in asteroids, Meteorit. Planet. Sci., 32, 349-363.

Kokubo, E. and S. Ida, (1995). Orbital evolution of protoplanets embedded in a swarm of planetesimals, Icarus, 114, 247-257.

Kokubo, E. and S. Ida, (1996). On Runaway Growth of Planetesimals, Icarus, 123, 180-191.

Kokubo, E. and S. Ida, (1998). Oligarchic Growth of Protoplanets, Icarus, 131, 171-178. 
Kokubo, E. and S. Ida, (2000). Formation of protoplanets from planetesimals in the solar nebula, Icarus $143,15-27$.

Kraus, R. G., L. E. Senft, and S. T. Stewart, (2011). Impacts onto H2O Ice: Scaling Laws for Melting, Vaporization, Excavation, and Final Crater Size, Icarus, 214, 724-738.

Matsui, T., (1993). Early evolution of terrestrial planets: Accretion, atmospheric formation, and thermal history, in Premitive Solar Nebula and Origin of Planets, Edt. H. Oya, pp 545559, Terra Scientific Publication Company, Tokyo.

McQueen, R. G., S. P. Marsh, J. W. Taylor, J. N. Fritz, and W. J. Carter (1970). The equation of state of solids from shock wave studies. In Kinslow (Ed.), High Velocity Impact Phenomena, Academic, New York, pp. 294-491.

McQueen, R.G., Marsh, S.P., Fritz, J.N., 1967. Hugoniot equation of state of twelve rocks. J. Geophys. Res. 72, 4999-5036.Melosh, H. J. 1989. Impact Cratering: A Geologic Process, Oxford Univ. Press, New York.

Melosh H. J. (1979), Acoustic fluidization: A new geologic process? J. Geophys. Res., 84, 75137520.

Melosh, M. J. (1989), Impact Cratering: A Geological Process, 1st ed., Oxford Univ. Press, New York.

Mitani, N. K. (2003), Numerical simulations of shock attenuation in solids and reevaluation of scaling law, J. Geophys. Res., 108, (E1), 5003, doi:10.1029/2000JE001472.

Monteux, J., N. Coltice, F. Dubuffet, Y. Ricard (2007), Thermo-mechanical adjustment after impacts during planetary growth, Geophys. Res. Lett, 34, L24201, doi:10.1029/2007GL031635

Monteux, J., Y. Ricard, N. Coltice, F. Dubuffet, M. Ulvrova (2009), A model of Metal-Silicate separation on Growing Planets, Earth and Planet. Sci. Lett., 287, 353-362, doi:10.1016/j.eps1.2009.08.020

Monteux, J., M. Jellinek, C .J., Johnson, (2013) Dynamics of core merging after a martian megaimpact, Icarus, 226, Issue 1, Pages 20-32, doi:10.1016/j.icarus.2013.05.008

Monteux, J., G., Tobie, G., Choblet, M., Le Feuvre (2014), Can large icy Moons accrete undifferentiated?, Icarus, 237, 15 July 2014, Pages 377-387, doi:10.1016/j.icarus.2014.04.041 
Monteux, J., Amit, H., Choblet, G., Langlais, B., Tobie, G. (2015) Giant impacts, heterogeneous mantle heating and a past hemispheric dynamo on Mars, Phys. Earth Planet. Int., 240, 114-124 doi:10.1016/j.pepi.2014.12.005.

O'Keefe, J. D., and T. J. Ahrens (1977), Impact-induced energy partitioning, melting, and vaporization on terrestrial planets, Lunar Planet. Sci. Conf., 8th, 3357-3374.

Perret, W.R., and R.C. Bass, (1975). Free-Field ground motion induced by underground explosions. Sandia Report SAND74-0252.

Pierazzo, E., Vickery, A.M., and Melosh, H.J., (1997). A re evaluation of impact melt production. Icarus 127, 408-423.

Pierazzo, E., Artemieva, N., Asphaug, E., Baldwin, E.C., Cazamias, J., Coker, R., Collins, G.S., Crawford, D.a., Davison, T., Elbeshausen, D., Holsapple, K.a., Housen, K.R., Korycansky, D.G., Wünnemann, K., (2008), Validation of numerical codes for impact and explosion cratering: impacts on strengthless and metal targets. Meteorit. Planet. Sci. 43 (12), 1917-1938.

Potter, R., Kring, D.A., Collins, G.S., Kiefer, W., McGovern, P., (2012). Estimating transient crater size using the crustal annular bulge: Insights from numerical modeling of lunar basin-scale impacts. Geophys. Res. Lett. 39, L18203

Rafikov, R.R., (2003). The growth of planetary embryos: Orderly, runaway, or oligarchic, The Astron. J., 125, 942-961.

Raymond, S.N., T. Quinn, and J. I. Lunine, (2006). High-resolution simulations of the final assembly of Earth-like planets, Terrestrial accretion and dynamics, Icarus 183, 265-282.

Ricard, Y., Srámek, O., Dubuffet, F., (2009). A multi-phase model of runaway core-mantle segregation in planetary embryos. Earth Planet Sci. Lett. 284, 144-150.

Roberts, J.H., and J. Arkani-Hamed, (2012). Impact-induced mantle dynamics on Mars, Icarus $218,278-289$.

Roberts, J.H., and J. Arkani-Hamed, (2014). Impact heating and coupled core cooling and mantle dynamics on Mars, J. Geophys. Res. Planets, 119, 729-744, doi:10.1002/2013JE004603.

Roberts, J.H., R.J. Lillis, and M. Manga (2009). Giant impacts on early Mars and the cessation of the Martian dynamo. J. Geophys. Res. 114, E04009. 
Schenk, P. et al. (2012), The Geologically Recent Impact Basins at Vesta's South Pole, Science, 336, 694-697.

Senshu, H., K. Kuramoto, and T. Matsui, (2002). Thermal evolution of a growing Mars, J. Geophys. Res., 107, E12, 5118, doi:10.1029/2001JE001819.

Schmidt, R. M., and K. R. Housen (1987), Some recent advances in the scaling of impact an explosion cratering, Int. J. Impact Eng., 5, 543- 560.

Thompson, S., Lauson, H., 1972. Improvements in the CHART D Radiation-hydrodynamic Code III: Revised Analytic Equations of State. Sandia National Laboratory Report SC-RR-71 0714, 113p.

Tonks, W.B., and H.J. Melosh, (1992). Core Formation by Giant Impacts, Icarus 100, 326-346.

Tonks, W.B., and H.J. Melosh, (1993). Magma Ocean Formation Due to Giant Impacts, J. Geophys. Res., 98, 5319-5333.

Trunin, R.F., 2001. Experimental Data on Shock Compression and Adiabatic Expansion of Condensed Matter. RFNC-VNIIEF, Sarov, p. 446, ISBN 5-85165-624-7.

Watters W.A., Zuber, M.T., Hager, B.H. (2009). Thermal perturbations caused by large impacts and consequences for mantle convection, J. Geophys. Res., 114, E02001

Wetherill, G.W., Inaba, S., (2000). Planetary accumulation with a continuous supply of planetesimals, Space Sci. Rev. 92, 311-320].

Wetherill, G.W., and G. R. Stewart, (1989). Accumulation of a Swarm of Small Planetesimals, Icarus 77, 330-357.

Wünnemann K., and B.A. Ivanov (2003). Numerical modelling of impact crater depth-diameter dependence in an acoustically fluidized target, Planet. and Space Sci., 51, 831-845.

Wünnemann K., G.S., Collins, and H. J. Melosh (2006). A strain-based porosity model for use in hydrocode simulations of impacts and implications for transient-crater growth in porous targets, Icarus 180, 514-52. 
581

582

583 Table 1. Typical parameter values for numerical hydrocode models

584

\begin{tabular}{lll}
\hline Target radius & $R$ & $3400 \mathrm{~km}$ \\
Target core radius & $R_{\text {core }}$ & $1700 \mathrm{~km}$ \\
Silicate mantle thickness & $\delta_{\mathrm{m}}$ & $1700 \mathrm{~km}$ \\
Impactor radius & $R_{\mathrm{imp}}$ & $50-200 \mathrm{~km}$ \\
Impact velocity & $V_{\mathrm{imp}}$ & $4-10 \mathrm{~km} / \mathrm{s}$ \\
\hline
\end{tabular}

Mantle properties (Silicates)

Initial density

$\rho_{\mathrm{m}}$

Equation of state type

Poisson

Strength Model

(iSALE parameters)

Acoustic Fluidization Model

(iSALE parameters)

Damage Model

(iSALE parameters)

Thermal softening and

porosity models

Core properties (Iron)

Initial density

$\rho_{\mathrm{c}} \quad 7840 \mathrm{~kg} / \mathrm{m}^{3}$

Equation of state type

Poisson

Strength Model

(iSALE parameters)

Acoustic Fluidization Model

(iSALE parameters)

Damage, thermal softening

and porosity models
$3314 \mathrm{~kg} / \mathrm{m}^{3}$

ANEOS

0.25

Rock

$\left(\mathrm{Y}_{\mathrm{i} 0}=10 \mathrm{MPa}, \mu_{\mathrm{i}}=1.2, \mathrm{Y}_{\mathrm{im}}=3.5 \mathrm{GPa}\right)$

Block

$\left(\mathrm{t}_{\mathrm{off}}=16 \mathrm{~s}, \mathrm{c}_{\mathrm{vib}}=0.1 \mathrm{~m} / \mathrm{s}, \mathrm{vib}_{\max }=200 \mathrm{~m} / \mathrm{s}\right)$

Ivanov

$\left(\varepsilon_{\mathrm{fb}}=10^{-4}, \mathrm{~B}=10^{-11}, \mathrm{p}_{\mathrm{c}}=3 \times 10^{8} \mathrm{~Pa}\right)$

None

\section{ANEOS}

0.3

Von Mises

$\left(\mathrm{Y}_{0}=100 \mathrm{MPa}\right)$

Block

$\left(\mathrm{t}_{\mathrm{off}}=16 \mathrm{~s}, \mathrm{c}_{\mathrm{vib}}=0.1 \mathrm{~m} / \mathrm{s}, \mathrm{vib}_{\max }=200 \mathrm{~m} / \mathrm{s}\right)$

None 
Table 2. Parameters of the peak shock pressure distribution and the corresponding particle velocity in the mantle of the Mars size model planet. The pressure is expressed as:

$\log (P)=a+n \log \left(d / R_{\text {imp }}\right)$

and the particle velocity as:

$$
\log \left(V_{\mathrm{p}}\right)=c+m \log \left(d / R_{\mathrm{imp}}\right)
$$

where the pressure $P$ is in GPa, the particle velocity $V_{p}$ is in $\mathrm{km} / \mathrm{s}, d$ is the distance from the impact site at the surface, and $R_{\text {imp }}$ is the impactor radius. $a$ and $c$ are the logarithm of pressure and particle velocity at the distance $R_{\text {imp }}$ from the impact site, and $n$ and $m$ are the decay exponents of pressure and particle velocity with distance from the impact site. $a, c, n$ and $m$ are

602

603

604 impact velocity dependent:

\section{5}

$a=\alpha+\beta \log \left(V_{\mathrm{imp}}\right)$

$n=\lambda+\delta \log \left(V_{\mathrm{imp}}\right)$

606

607

608

609

610

A misfit value is obtained by calculating the standard deviation of a line fitted to the hydrocode data within a given region: $\epsilon=1 / \mathrm{N} \sqrt{\sum_{1}^{N}\left(Y_{\text {data }}-Y_{\text {regression }}\right)^{2}}$, where $N$ is the total number of points, $Y_{\text {data }}$ is the hydrocode result and $Y_{\text {regression }}$ denotes the value obtained by the linear regression. The zero misfit implies that the regression line is fitted to only 2 points, hence an exact fitting.

$V_{\mathrm{imp}} \quad a$ $(\mathrm{km} / \mathrm{s})$

\section{Near Field}

$n$

$$
-0.4530
$$

$-0.6296$

$-0.4713$

$-0.3237$

$-0.3302$

$-0.1228$

$-0.1364$ misfit

1.074E-07

4.616E-03

$8.429 \mathrm{E}-08$

$5.960 \mathrm{E}-08$

$5.960 \mathrm{E}-08$

8.429E-08

1.332E-07 $c$

0.1276

0.2901

0.3722

0.3315

0.5736

0.6817

0.7354 $m$

$-1.1132$

$-1.0437$

$-0.8573$

$-0.4286$

$-0.6567$

$-0.2156$

$-0.2622$ misfit

3.071E-08

$6.6837 \mathrm{E}-04$

0.000

$1.490 \mathrm{E}-08$

0.000

4.214E-08

8.411E-03

$626 \alpha=0.040, \quad \beta=1.914, \lambda=-1.214, \delta=1.058$

$627 \gamma=-0.795, \Omega=1.502 \quad \eta=-2.602, \zeta=2.368$

628

629 


\begin{tabular}{|c|c|c|c|c|c|c|c|}
\hline 630 & & & Mid Fielc & & & & \\
\hline 631 & $V_{\mathrm{imp}}$ & $a$ & $n$ & misfit & $c$ & $m$ & misfit \\
\hline 632 & $(\mathrm{~km} / \mathrm{s})$ & & & & & & \\
\hline 633 & 4 & 1.3714 & -0.9459 & $2.0576 \mathrm{E}-03$ & 0.0917 & -1.0211 & $3.8861 \mathrm{E}-04$ \\
\hline 634 & 5 & 1.5978 & -0.9995 & $6.5599 \mathrm{E}-04$ & 0.2911 & -1.0402 & 7.3517E-04 \\
\hline 635 & 6 & 1.6367 & -0.8038 & $4.7266 \mathrm{E}-03$ & 0.4143 & -0.9942 & 8.0339E-04 \\
\hline 636 & 7 & 1.8821 & -0.9792 & $2.8677 \mathrm{E}-03$ & 0.5141 & -0.9563 & $1.8048 \mathrm{E}-03$ \\
\hline 637 & 8 & 1.9735 & -0.8576 & $3.5979 \mathrm{E}-03$ & 0.6750 & -0.9888 & $6.5566 \mathrm{E}-05$ \\
\hline 638 & 9 & 2.0060 & -0.7072 & $6.2120 \mathrm{E}-03$ & 0.8611 & -1.1259 & $2.0130 \mathrm{E}-03$ \\
\hline 639 & 10 & 2.0224 & -0.6059 & 8.2497E-03 & 0.9317 & -1.1190 & $1.3516 \mathrm{E}-03$ \\
\hline 640 & $\alpha=0.3$ & $346, \quad \beta=$ & $6, \quad \lambda=-1$ & $\delta=0.768$ & & & \\
\hline 641 & $\gamma=-1.2$ & 206, $\Omega=$ & $\eta=-0$ & $\zeta=-0.208$ & & & \\
\hline 642 & & & & & & & \\
\hline 643 & & & & & & & \\
\hline 644 & & & Far Field & & & & \\
\hline 645 & $V_{\mathrm{imp}}$ & $a$ & $n$ & misfit & $c$ & $m$ & misfit \\
\hline 646 & $(\mathrm{~km} / \mathrm{s})$ & & & & & & \\
\hline 647 & 4 & 1.5136 & -1.1453 & $6.4139 \mathrm{E}-04$ & 0.2397 & -1.2158 & $1.1549 \mathrm{E}-03$ \\
\hline 648 & 5 & 1.7356 & -1.1640 & $5.0752 \mathrm{E}-04$ & 0.4635 & -1.2389 & $1.0468 \mathrm{E}-03$ \\
\hline 649 & 6 & 1.9107 & -1.1864 & $6.9751 \mathrm{E}-04$ & 0.6248 & -1.2531 & $1.2862 \mathrm{E}-03$ \\
\hline 650 & 7 & 2.0602 & -1.2182 & $6.0862 \mathrm{E}-04$ & 0.7628 & -1.2783 & $1.3663 \mathrm{E}-03$ \\
\hline 651 & 8 & 2.2186 & -1.2816 & 3.3932E-04 & 0.8936 & -1.3220 & $1.0471 \mathrm{E}-03$ \\
\hline 652 & 9 & 2.4057 & -1.3818 & 1.1730E-03 & 1.0620 & -1.4091 & $8.7868 \mathrm{E}-04$ \\
\hline 653 & 10 & 2.5440 & -1.4492 & $1.5957 \mathrm{E}-03$ & 1.1887 & -1.4687 & 1.3772E-03 \\
\hline 654 & $\alpha=-0$. & $056, \beta=$ & $58, \lambda=-0$ & $\delta=-0.744$ & & & \\
\hline 655 & $\gamma=-1.1$ & 177, $\Omega=$ & $33, \quad \eta=-0$ & $\zeta=-0.600$ & & & \\
\hline 656 & & & & & & & \\
\hline 657 & & & & & & & \\
\hline 658 & & & & & & & \\
\hline 659 & & & & & & & \\
\hline 660 & & & & & & & \\
\hline 661 & & & & & & & \\
\hline 662 & & & & & & & \\
\hline 663 & & & & & & & \\
\hline 664 & & & & & & & \\
\hline 665 & & & & & & & \\
\hline 666 & & & & & & & \\
\hline 667 & & & & & & & \\
\hline 668 & & & & & & & \\
\hline 669 & & & & & & & \\
\hline 670 & & & & & & & \\
\hline 671 & & & & & & & \\
\hline 672 & & & & & & & \\
\hline 673 & & & & & & & \\
\hline 674 & & & & & & & \\
\hline 675 & & & & & & & \\
\hline
\end{tabular}


676

677

678

679

680

681

682

683

684

685

686
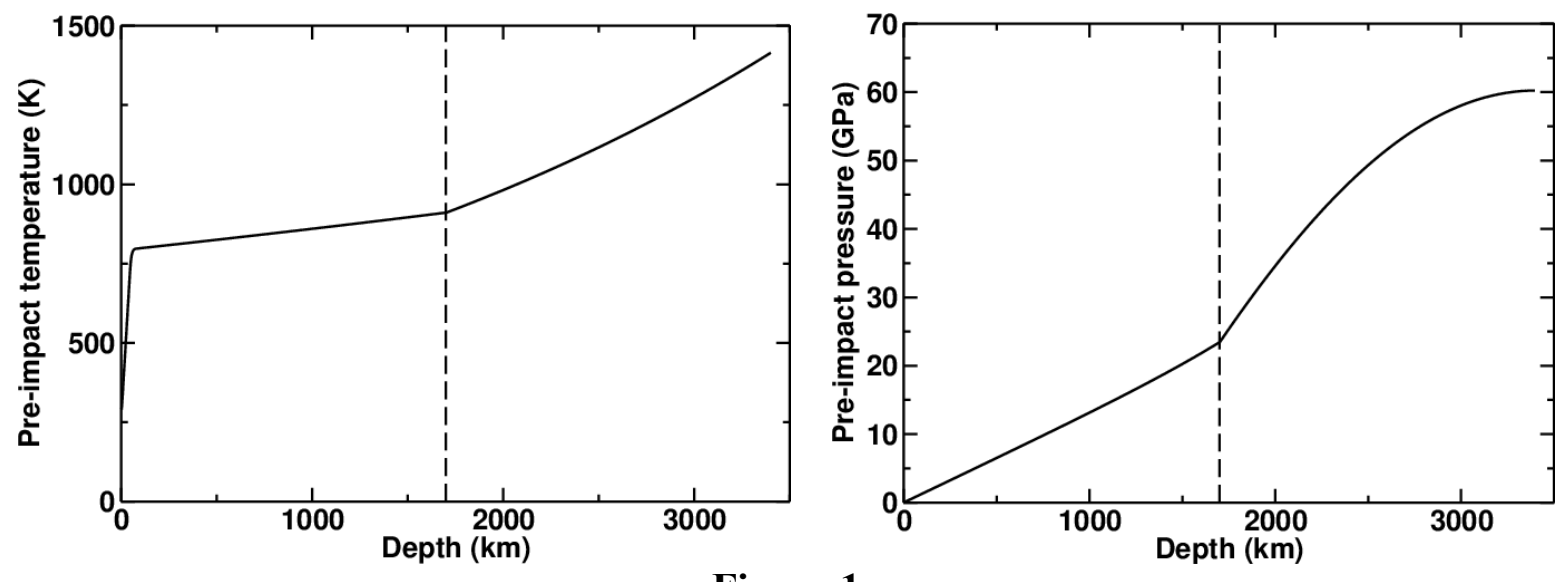

Figure 1

Figure 1: Pre-impact temperature (left) and lithostatic pressure (right) as a function of depth. The dashed lines illustrate the core-mantle boundary. 

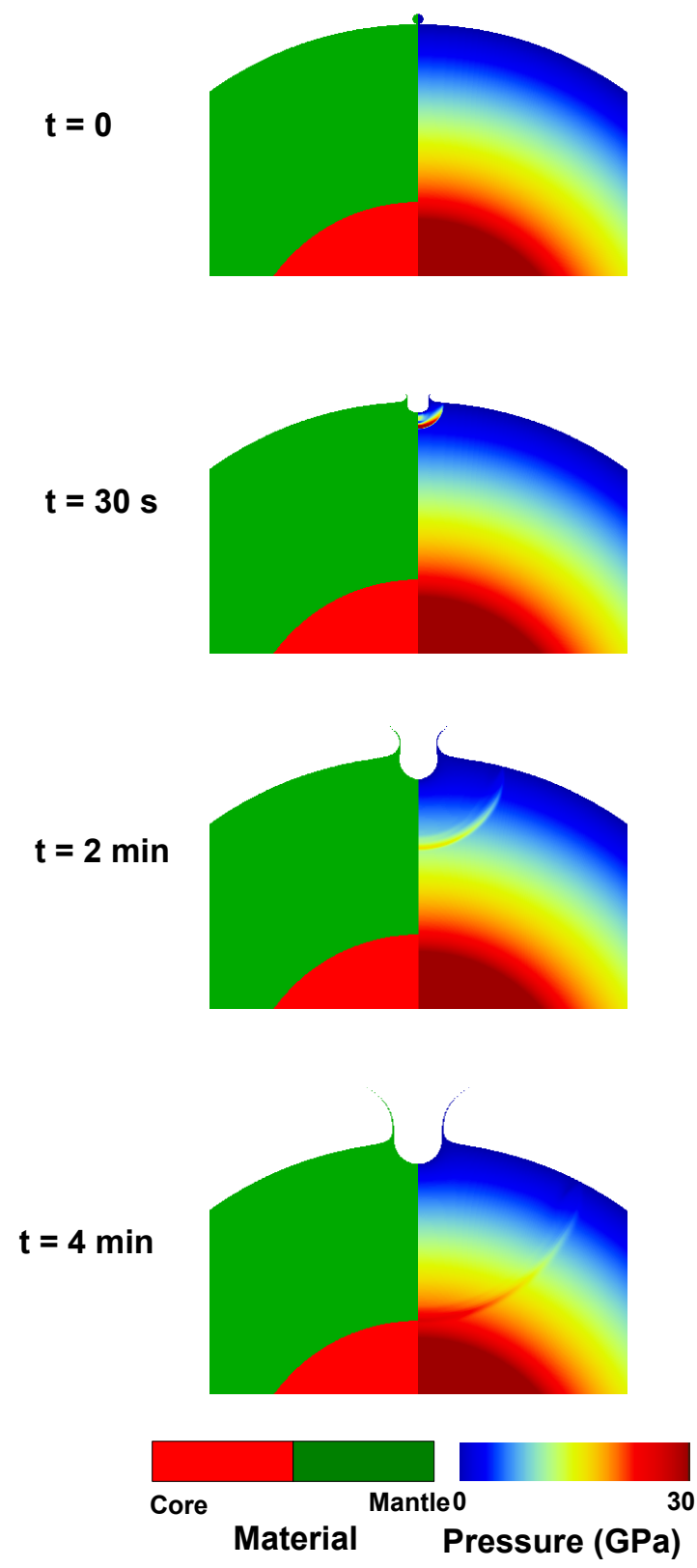

Figure 2: A close up view of the material repartition (left column) and total pressure (right column) as functions of time (from top to bottom) in the model planet (for $V_{\mathrm{imp}}=10 \mathrm{~km} / \mathrm{s}$ and $D_{\text {imp }}=100 \mathrm{~km}$ ). In this model, the grid resolution is $2 \mathrm{~km}$ in all directions. The silicate mantle 691 and the impactor are made of dunite. 


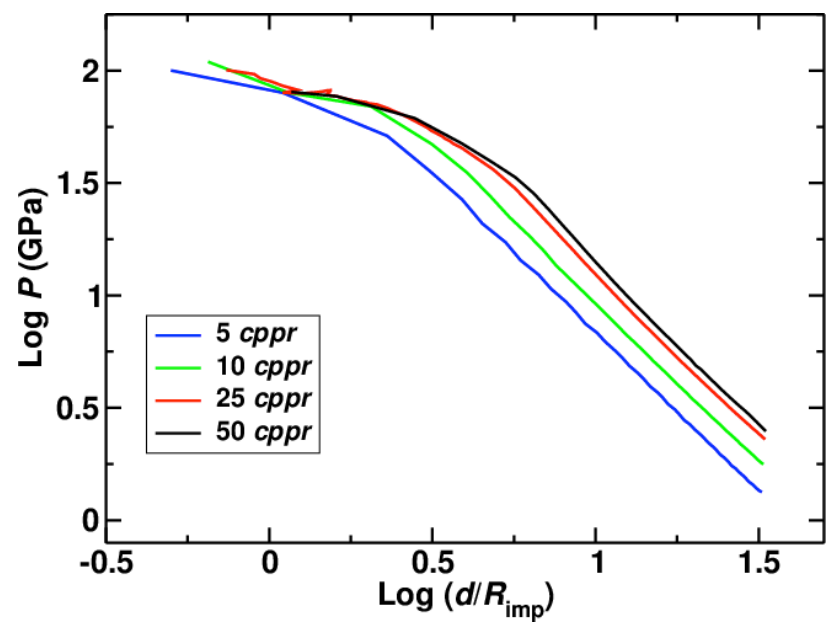

Figure 3a

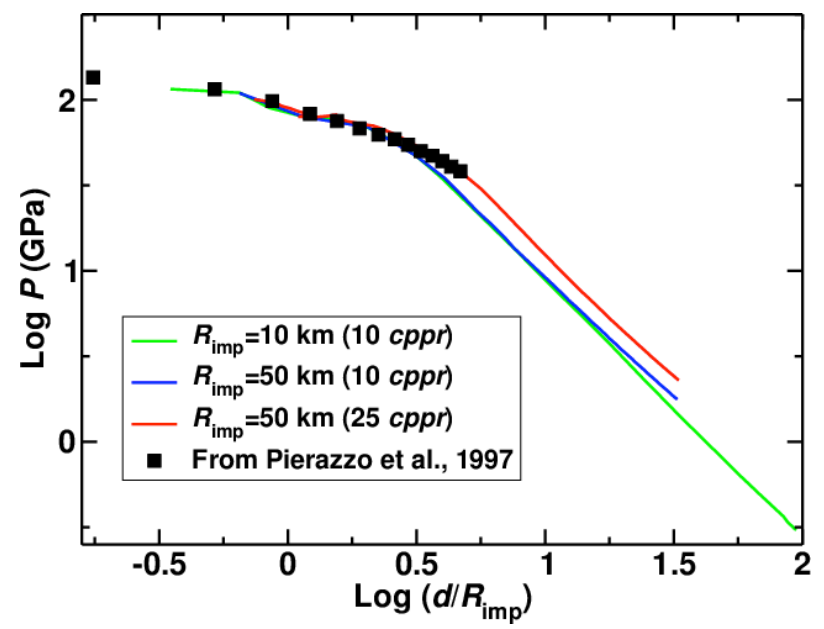

Figure 3b

Figure 3: Peak pressure decrease as a function of depth normalized by the radius of the impactor for the impact velocity of $10 \mathrm{~km} / \mathrm{s}$. The silicate mantle as well as the impactor are made of dunite. 3a: Influence of the spatial resolution. Here we only consider the case with $R_{\text {imp }}=50 \mathrm{~km}$. The results from our hydrocode models are shown by colored curves with a spatial resolution ranging from 5 to $50 \mathrm{cppr}$. 3b: Comparison of our results with $R_{\text {imp }}=50 \mathrm{~km}$ (red curve, $25 \mathrm{cppr}$ ) and $R_{\text {imp }}=10 \mathrm{~km}$ (green curve, $10 \mathrm{cppr}$ ) with the results from a similar model of Pierazzo et al., (1997) (black squares). 


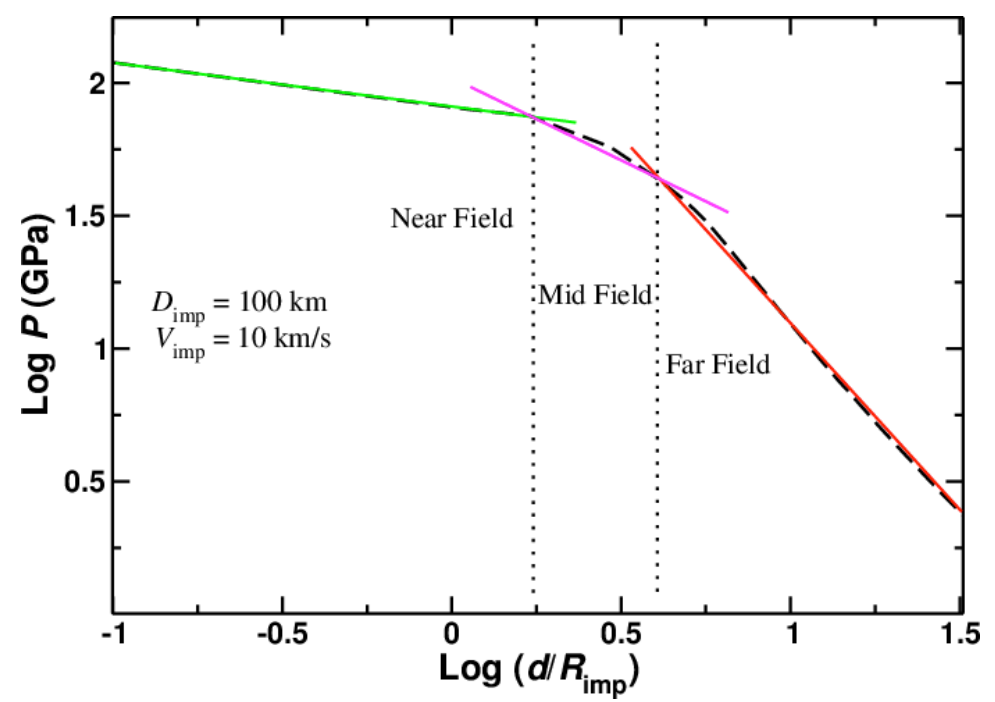

728 Figure 4. Shock pressure versus normalized distance from the impact site at the surface

729 produced by a $100 \mathrm{~km}$ diameter impactor with an impact velocity of $10 \mathrm{~km} / \mathrm{s}$. The dashed curve

730 represents the hydrocode model, while the straight lines are fitted to three different parts of the 731 hydrocode model. 
735

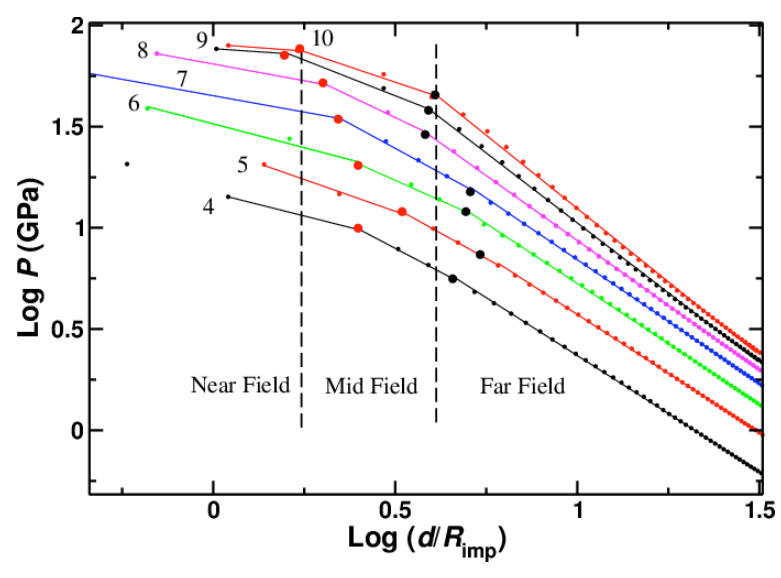

Figure 5a

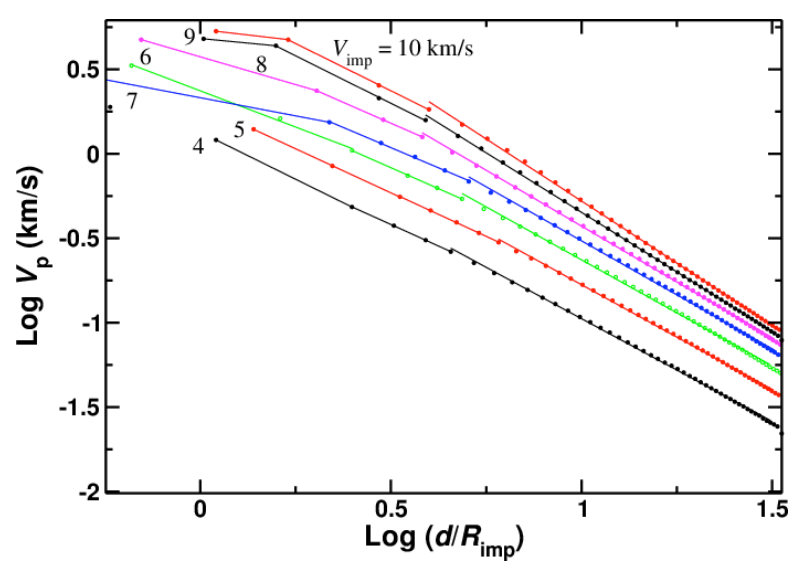

Figure 5b

Figure 5a. Shock pressure versus normalized distance from the impact site at the surface for an impactor of $100 \mathrm{~km}$ diameter and impact velocities ranging from 4 to $10 \mathrm{~km} / \mathrm{s}$. The numbers on the curves are the impact velocities. The hydrocode results are presented by dots, while the regression lines to the near field, mid field and far field regions are straight lines. The larger dots show the intersections of the linear regression lines. For example a dot that separates near field from mid field is the intersection of the regression lines fitted to the near field and mid field data. 5b. shows the corresponding particle velocity. 
747

748

749

750

751

752

753

754

755

756

757

758

759

760

761

762

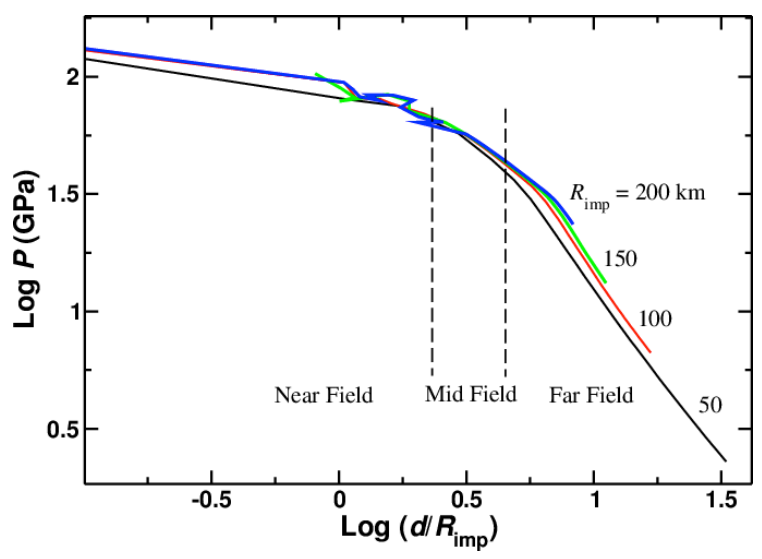

Figure 6a

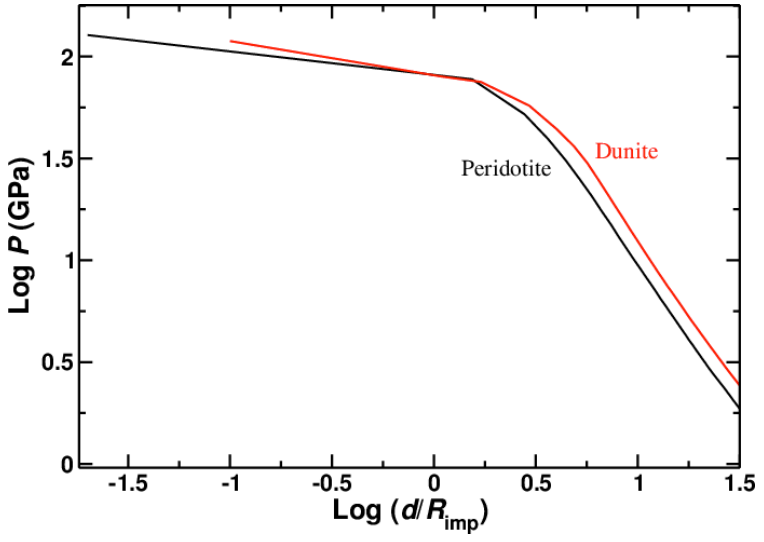

Figure 6b

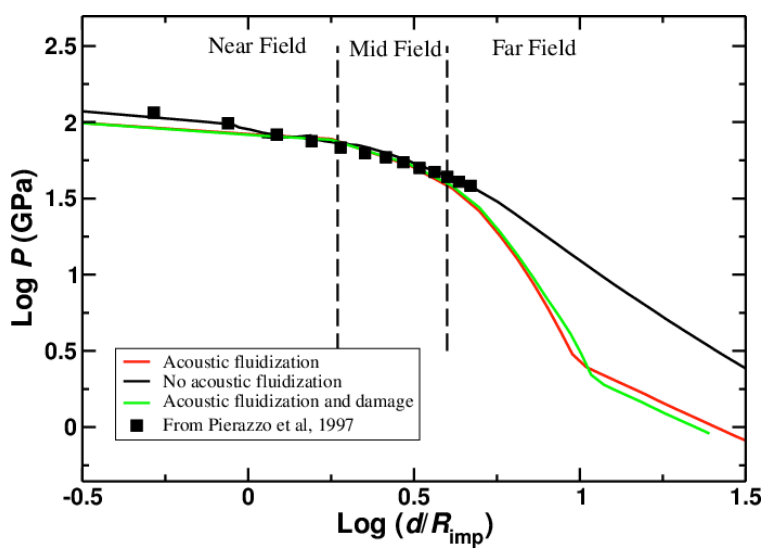

Figure 6c

Figure 6a. shows the hydrocode results for impactors of 50 to $400 \mathrm{~km}$ diameter and impact velocity of $10 \mathrm{~km} / \mathrm{s}$. 6b. compares the hydrocode results using dunite and peridotite as mantle rock types, for an impactor of $100 \mathrm{~km}$ diameter and impact velocity of $10 \mathrm{~km} / \mathrm{s}$. $6 \mathbf{c}$ illustrates the shock pressure as a function of $d / R_{\text {imp }}$ with (red curve) and without (black curve) acoustic fluidization. The green curve represents the results considering an Ivanov damage model. (For comparison, the black squares represent the results from Pierazzo et al., (1997], which has not considered acoustic fluidization). 
765

766

767

768

769

770

771

772

773

774

775

776

74

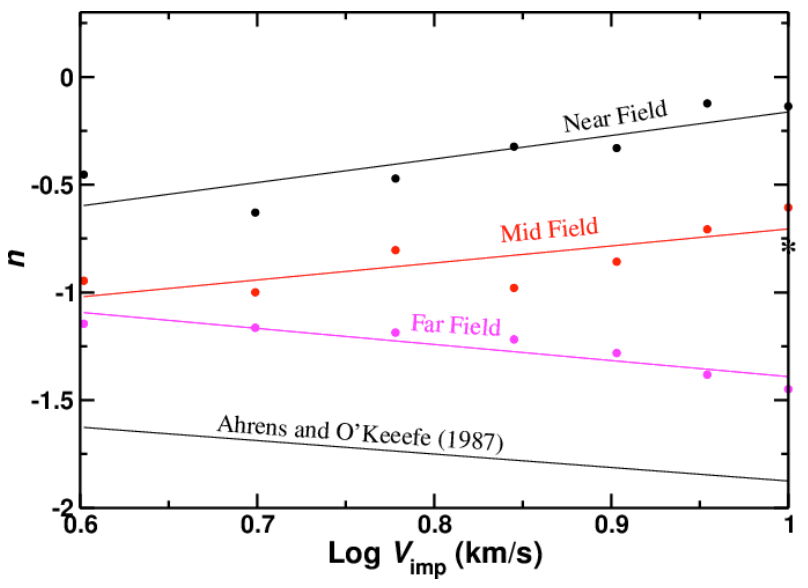

Figure $7 b$

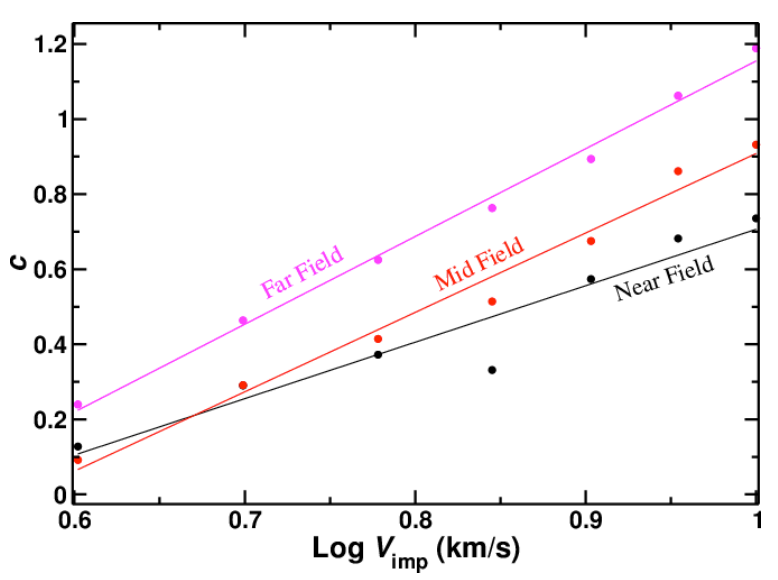

Figure 7c

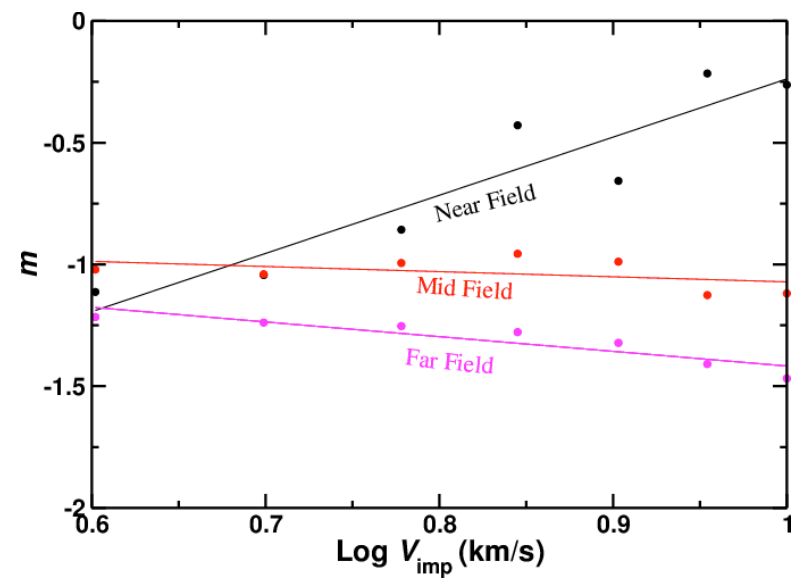

Figure 7d

Figure 7. Dependence of regression parameters $a, n, c$, and $m$ from Eq. 4 and 5 on the impact

72 velocity. Dots are based on hydrocode models and lines are regression fits, see Table 2 


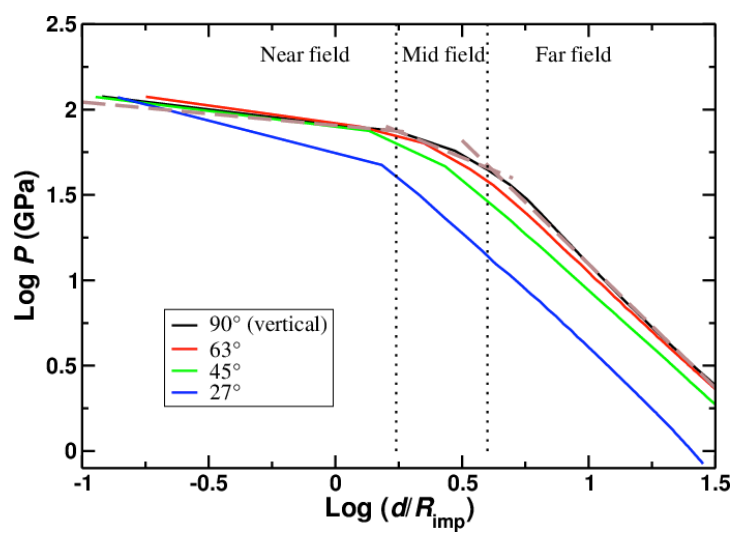

Figure 8a

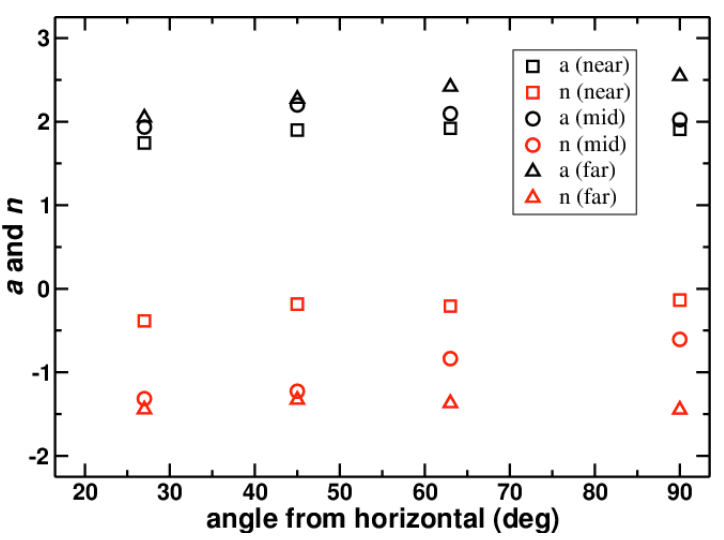

Figure 8b

779 Figure 8. The shockwave propagation as a function of the angle from the horizontal for the

780 classical case $\left(V_{\mathrm{imp}}=10 \mathrm{~km} / \mathrm{s}\right.$ and $\left.R_{\mathrm{imp}}=50 \mathrm{~km}\right) .8 \mathbf{a}$ Shock pressure decrease as a function of the

781 distance to the impact site for propagation angles ranging between $27^{\circ}$ and $90^{\circ}$. $8 \mathbf{b}$ Dependence 782 of regression parameters $a$ and $n$ (from Eq. 4 and 5) on the angle from horizontal. 

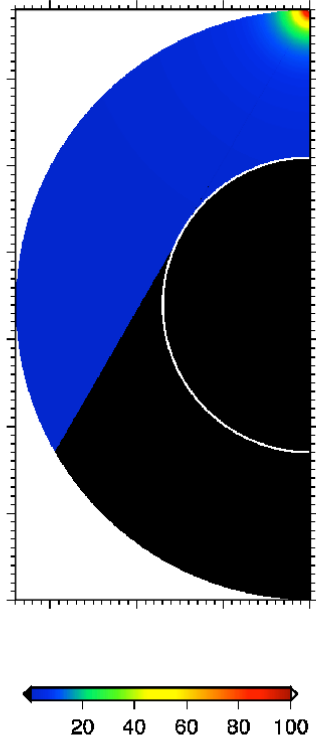

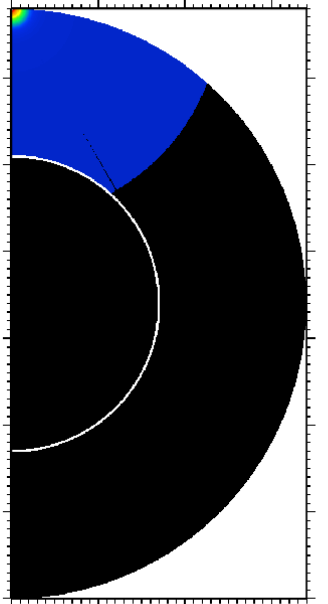

2004006008001000

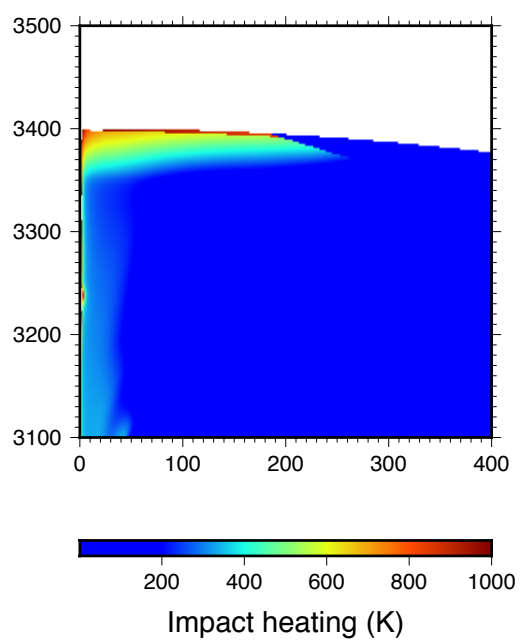

Impact heating $(\mathrm{K})$

Peak shock pressure (GPa) Shock temperature (K)

Figure 9: $2 \mathrm{D}$ distribution of the peak shock pressure (left panel), and corresponding shock induced temperature increase (central panel) determined from our scaling laws for an impactor of $100 \mathrm{~km}$ in diameter and an impact velocity of $10 \mathrm{~km} / \mathrm{s}$ calculated using the scaling laws in near field, mid field, and far field and the impact-induced temperature increase model from Watters et al. [2009] (See Eq. 7-9). The parameter values are based on regression lines from Figure 7 and Table 2. The grid spacing is $2 \mathrm{~km}$ in radial direction and 0.03 degrees in the colatitude direction. Only direct shock wave is considered. The black region in the mantle does not receive direct waves. The thin line extending from the impact site at the surface is the tangent line to the core. It shows that the part of the core receiving direct shock wave is not more than $25 \%$. The color is saturated at higher values to better illustrate the regions with lower values. The right panel shows a close-up view of the corresponding temperature increase obtained from iSALE more than 30 minutes after the impact and illustrates the modifications that occur during the late stages of the impact process (excavation, central peak formation, slumping...). However, the intensity of impact heating is in agreement with our scaling laws. 


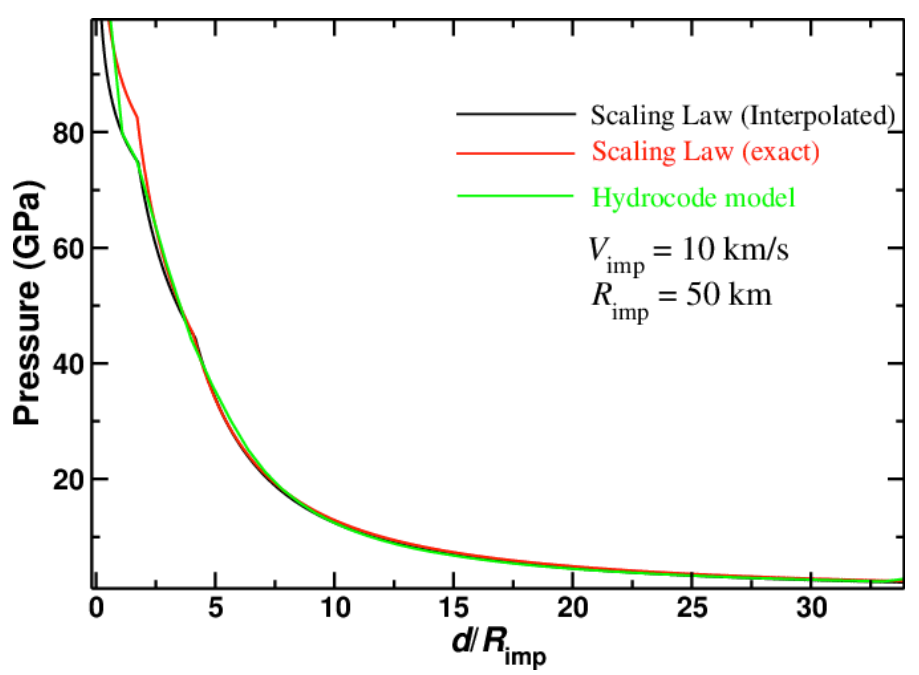

809 Figure 10. Shock pressure versus distance from the impact site normalized to the impactor

810 radius. The hydrocode model is the output of the hydrocode simulation. In the exact model we

811 have used $a$ and $n$, while $\alpha, \beta, \lambda$ and $\delta$ are used in the interpolated model, see Table 2.

812

813

814

815

816

817

818

819 


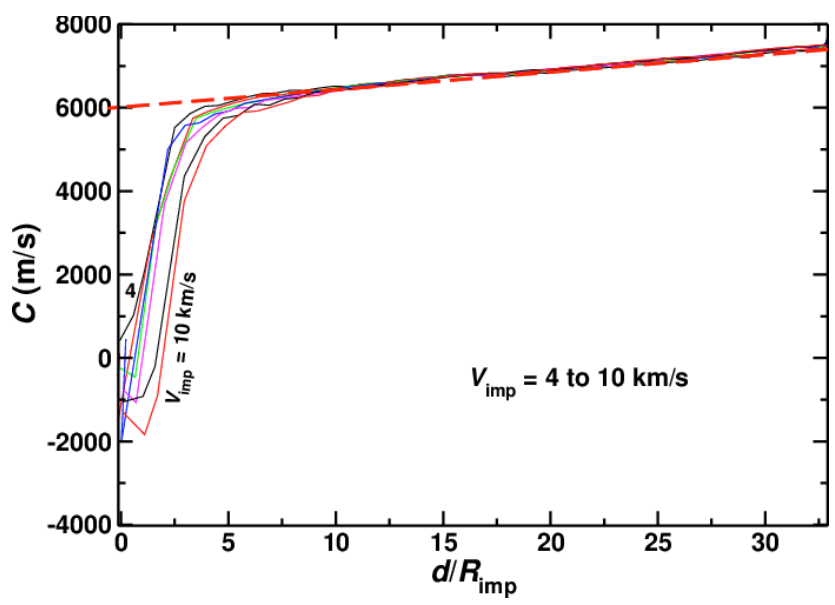

821 Figure 11. The acoustic velocity determined using Equation 10 for an impactor of $100 \mathrm{~km}$

822 diameter, and impact velocities ranging from 4 to $10 \mathrm{~km} / \mathrm{s}$. The $S$ parameter in the equation is set 823 to 1.2 . The red dashed line is a line fit to $C$ in the deeper region. 